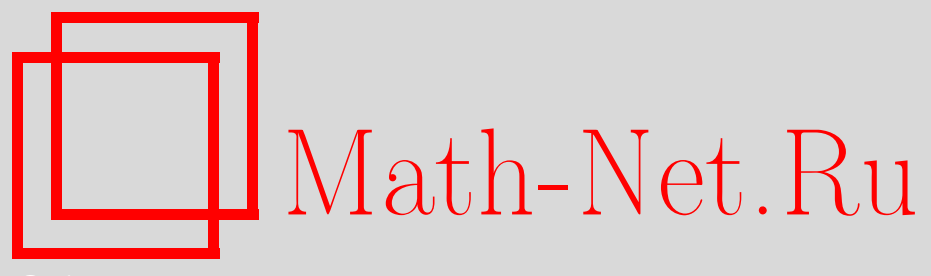

А. И. Аптекарев, Д. Н. Туляков, Асимптотические режимы в зоне насыщения для C-D-ядер ансамбля ортогональных многочленов Мейкснера, УМН, 2011, том 66, выпуск 1, 181-182

DOI: https://doi.org/10.4213/rm9409

Использование Общероссийского математического портала Math-Net.Ru подразумевает, что вы прочитали и согласны с пользовательским соглашением http://www . mathnet.ru/rus/agreement

Параметры загрузки:

IP : 52.205 .19 .152

26 апреля 2023 г., 14:11:27

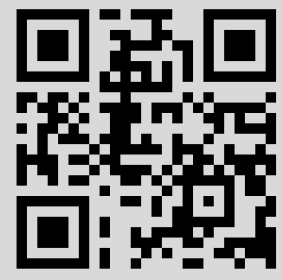




\title{
Асимптотические режимы в зоне насыщения для C-D-ядер ансамбля ортогональных многочленов Мейкснера
}

\author{
А. И. Аптекарев, Д. Н. Туляков
}

1. Пусть $\mathscr{X}$ - дискретное счетное подмножество $\mathbb{R}, W>0$ - весовая функция на $\mathscr{X}$. По определению (см. [1]), $n$-точечным ансамблем ортогональных многочленов $(\mathrm{OM})$ относительно веса $W$ называют случайные конфигурации $\left\{\vec{x}:=\left(x_{1}, \ldots, x_{n}\right)\right.$ : $\left.x_{1}>\cdots>x_{n}, x_{1}, \ldots, x_{n} \in \mathscr{X}\right\}$ с вероятностями $\operatorname{Prob}\left[\left(x_{1}, \ldots, x_{n}\right)\right]=Z_{n}^{-1} \Pi(\vec{x})$, где

$$
\Pi(\vec{x}):=\prod_{i=1}^{n} W\left(x_{i}\right) \prod_{1 \leqslant i<j \leqslant n}\left(x_{i}-x_{j}\right)^{2}, \quad Z_{n}:=\sum_{\vec{x}: x_{1}>\cdots>x_{n}} \Pi(\vec{x}) .
$$

Это название вызвано следующим обстоятельством. Если рассмотреть последовательность многочленов $\left\{Q_{k}\right\}$, являющихся ортогонализацией в $l^{2}(\mathscr{X}, W)$ последовательности $\left\{\tilde{x}^{k}\right\}$, и определить ядра Кристоффеля-Дарбу (C-D) с весом $W$

$$
K_{n}(\tilde{x}, \tilde{y}):=\sqrt{W(\tilde{x}) W(\tilde{y})} \sum_{i=0}^{n-1} \frac{Q_{i}(\tilde{x}) Q_{i}(\tilde{y})}{\left\|Q_{i}\right\|^{2}},
$$

то, как известно (см., например, [2]), вероятность того, что случайная конфигурация $\left(x_{1}, \ldots, x_{n}\right)$ содержит заданную $\left(y_{1}, \ldots, y_{k}\right)$, равна

$$
\operatorname{Prob}\left[\left(y_{1}, \ldots, y_{k}\right)\right]=\operatorname{det}\left[K_{n}\left(y_{i}, y_{j}\right)\right]_{i, j=1}^{k} .
$$

В настоящей заметке мы рассматриваем $n$-точечные ансамбли многочленов Мейкснера, ортогональных относительно дискретного веса:

$$
W_{\beta, \sigma}(\tilde{x}):=\frac{\Gamma(\beta+\tilde{x})}{\tilde{x} !} \sigma^{\tilde{x}}, \quad \tilde{x} \in \mathbb{Z}_{+}, \quad \beta>0, \quad 0<\sigma<1 .
$$

Наша цель - асимптотика (при $n \rightarrow \infty)$ C-D-ядер (2) для ансамбля Мейкснера (1), (3) при различных соотношениях между ростом $n$ и $\tilde{x}$.

2. Для упрощения последующих формул введем новые параметры и сделаем в определении веса Мейкснера (3) замену переменной $c:=\sigma^{-1}, b:=\beta-1, x:=\tilde{x}(c-1)-\beta$. В новых обозначениях многочлены Мейкснера $\left\{Q_{n}(x)\right\}$ ортогональны по дискретной мере $\mu(x):=\sum_{x_{0} \in(c-1) \mathbb{Z}_{+}-\beta} \rho(x) \delta\left(x-x_{0}\right)$, с весом

$$
\rho(x):=\frac{c^{-(b+1) /(c-1)}}{c-1} \frac{((x+c b+1) /(c-1)) !}{((x+b+1) /(c-1)) !} c^{-x /(c-1)}, \quad x \in \mathbb{R}_{+} .
$$

В работе [3] разработана общая методика построения асимптотических разложений для базисов решений рекуррентных соотношений в перекрывающихся областях плоскости $(n, x)$ и их согласования в переходных зонах. Мы применяем асимптотики многочленов Мейкснера, полученные в [3], для описания (в различных зонах плоскости $(n, x))$ предельных режимов C-D-ядер (2). По формуле Кристоффеля-Дарбу

$$
K_{n}(x, y)=\frac{\tilde{q}_{n}(x) \tilde{q}_{n-1}(y)-\tilde{q}_{n}(y) \tilde{q}_{n-1}(x)}{\left\|Q_{n-1}\right\|^{2}(x-y)}, \quad \tilde{q}_{n}(x):=\sqrt{\rho(x)} Q_{n}(x),
$$

где $\left\|Q_{n}\right\|^{2}=n !(n+b) ! c^{n}(c /(c-1))^{b+1}$. Используя непрерывное заполнение веса (4), мы будем рассматривать C-D-ядро (5) в любой точке соответствующей зоны.

Работа выполнена при поддержке РФФИ (гранты № 11-01-00245, 09-01-12024), программы "Ведущие научные школы" (грант НШ-8033.2010.1) и программы № 1 ОМН РАН; второй автор также поддержан грантами РФФИ № 09-01-12160, 10-01-00682. 
3. Между соседними массами меры ортогональности не может размещаться более одного нуля ОМ. Зоны, в которых каждый интервал между соседними массами занят нулем OM, называются зонами насыщения (saturation zone). Для многочленов Мейкснера эта зона характеризуется масштабом

$$
x=n t, \quad t \in K \Subset\left(0,(\sqrt{c}-1)^{2}\right), \quad n \rightarrow \infty .
$$

В зоне насыщения нули ОМ с экспоненциальной скоростью (при $n \rightarrow \infty$ ) притягиваются к точкам, где сосредоточены массы дискретной меры. В этой зоне уместно рассматривать различные асимптотические режимы для C-D-ядра, а именно режим (6) в окрестности произвольной точки зоны насыщения и режим в окрестности масс

$$
X_{k}=k(c-1)-b-c, \quad k \in \mathbb{Z}_{+},
$$

с экспоненциальным масштабированием при $t:=t_{k}:=X_{k} / n \in K \Subset\left(0,(\sqrt{c}-1)^{2}\right)$ :

$$
e^{-2 \Sigma(n, t)}:=\left(\frac{c+1-t+\sqrt{D}}{2 \sqrt{c}}\right)^{-2 n-b+1}\left(\frac{(c-1)^{2}-(c+1) t+(c-1) \sqrt{D}}{2 t \sqrt{c}}\right)^{2 k+b-1},
$$

где $D(t):=(c+1-t)^{2}-4 c$ и $n \rightarrow \infty$.

Теорема. Для $C$-D-ядра (5), равномерно по $\xi, \eta \in \widetilde{K} \Subset \mathbb{R}$, справедливо:

1) в режиме (6)

$$
\begin{aligned}
K_{n}(n t+\xi, n t+\eta)= & e^{(\xi+\eta) \Psi^{\prime}(t)} \sin \left(\pi \frac{n t+\xi+b+c}{c-1}\right) \sin \left(\pi \frac{n t+\eta+b+c}{c-1}\right) \frac{e^{2 n \Psi(t)}}{n} \\
& \times\left(\Phi(t)+O\left(n^{-1}\right)\right)+O\left(e^{\left|(\xi-\eta) \Psi^{\prime}(t)\right|}\right) ;
\end{aligned}
$$

2) в смешанном режиме (6) и (8) в окрестности точки массы (7)

$$
K_{n}\left(X_{k}+e^{-2 \Sigma(n, t)} n \xi, X_{k}+\eta\right)=\frac{\sin \frac{\pi \eta}{c-1}}{\eta} e^{\eta \Psi^{\prime}(t)}\left(\xi \eta \widetilde{\Phi}_{1}(t)+\widetilde{\Phi}_{2}(t)\right)+O\left(n^{-1}\right) ;
$$

3) в режиме (8) в окрестностях разных точек масс (7) $n р и ~ k-m=O(1)$

$$
\begin{aligned}
& K_{n}\left(X_{k}+e^{-2 \Sigma\left(n, t_{k}\right)} \xi, X_{m}+e^{-2 \Sigma\left(n, t_{m}\right)} \eta\right) \\
& \quad=(-1)^{k-m} \frac{e^{-\Sigma\left(n, t_{k}\right)-\Sigma\left(n, t_{m}\right)}}{k-m}\left((\xi-\eta) \widetilde{\Psi}\left(t_{k}, t_{m}\right)+O\left(n^{-1}\right)\right) .
\end{aligned}
$$

Здесъ $\Psi(t):=\operatorname{arccosh} \frac{c+1-t}{2 \sqrt{c}}-\frac{t}{c-1} \operatorname{arccosh} \frac{(c-1)^{2}-(c+1) t}{2 t \sqrt{c}}$, а функции $\Phi, \Phi_{1}$, $\Phi_{2}, \widetilde{\Psi}$ от $t$ явным образом выражаются через параметры веса Мейкснера с и $\beta$ ине зависят от $n$ и $\xi, \eta$.

\section{Список литературы}

[1] A. Borodin, G. Olshanskii, Mosc. Math. J., 6:4 (2006), 629-655. [2] P. Deift, Orthogonal polynomials and random matrices: a Riemann-Hilbert approach, New York Univ., Courant Institute of Mathematical Sciences, New York; Amer. Math. Soc., Providence, RI, 1999, ISBN: 0-9658703-2-4; 0-8218-2695-6, viii+273 pр. [3] Д. Н. Туляков, Матем. сб., 201:9 (2010), 111-158.

А. И. Аптекарев (А. I. Aptekarev)

Институт прикладной математики им. М. В. Келдыша РАН

E-mail: aptekaa@keldysh.ru

Д. Н. Туляков (D. N. Tulyakov)

Институт прикладной математики им. М. В. Келдыша РАН

E-mail: dnt@mail.nnov.ru
Представлено А. Г. Сергеевым Принято редколлегией 02.10 .2010 